\title{
Optimized WiMAX System over Fading Channels Using FEC Codes
}

\author{
Mahwash Manzoor and Lavish Kansal* \\ School of Electronics and Electrical Engineering, Lovely Professional University, India; \\ mahwash25@yahoo.com, lavish.15911@lpu.co.in
}

\begin{abstract}
The Wireless Interoperability Microwave Access (WiMAX) system in this paper makes use of various forward error correction codes to improve and hence optimize the performance of the system. Two diverse fading channels have been used to study the response of the WiMAX system based on Forward Error Correction (FEC) encoding and M-ary Phase Shift Keying (M-PSK) modulation. Since WiMAX is used for both Line Of Sight (LOS) and Non Line-Of-Sight (NLOS) communication so, Rayleigh and Rician channels have been used, out of which one has LOS component, and other has no LOS component. The performance of WiMAX system has been studied by analyzing the graphs between Bit Error Rate (BER) and Signal to Noise Ratio (SNR) for all the different cases. It is observed that out of all coding techniques reed Solomon (RS) encoding techniques performs better at lower order modulation schemes by providing the BER of $10^{-3}$ at lower values of SNR in comparison to all other FEC encoding techniques. But, at higher order modulation level turbo encoding scheme outperform all other FEC schemes. Also, out of channels rician channel has a better response because of one strong line of sight constituent in comparison to Rayleigh fading channel. Further, modified FEC encoding schemes can be incorporated to enhance the BER performance of the WiMAX system.
\end{abstract}

Keywords: BER, Fading, FEC, MPSK, WiMAX

\section{Introduction}

The WiMAX has had explosive growth in recent years as a broadband communication technology which serves people with high transmission rates and large coverage areas compared to any of its opponents. It was initially developed in the year 1998 by National Institute of Standards and Technology (NIST) and then handed over to the working group of IEEE 802.16. It can be fixedwhich is standardized as IEEE-802.16d or mobile-which is standardized to IEEE-802.16e. For it to meet the current demands of speed, the probability of error should be insignificant ${ }^{1}$. The growth of multimedia communication puts the burden on wireless communication systems for higher speeds and greater bandwidth. In wired communication, it may result in huge investment on physical connections between remote locations but in the case of wireless communication, the implementation cost is cut down due to no tangible connections required between remote locations. This comes with a disadvantage of changing statistical nature of radio and wireless channel and hence performance degradation. WiMAX is the solution for all problems and is rising as a $4 \mathrm{G}$ technology

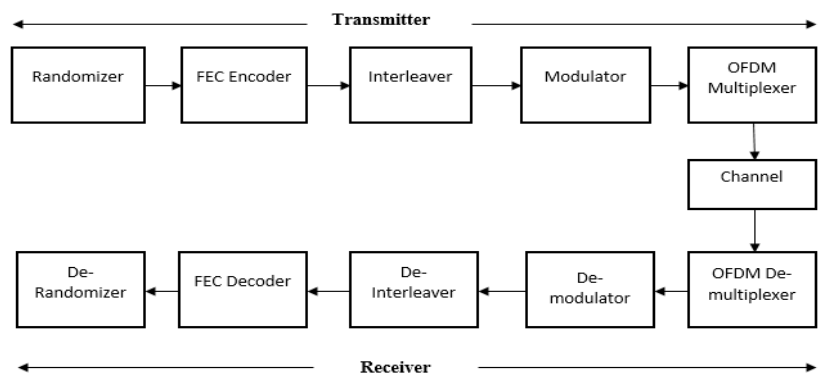

Figure 1. Physical layer of WiMAX

with Non NLOS transmission in the frequency range of $2 \mathrm{GHz}$ to $11 \mathrm{GHz}$ and Line-Of-Sight (LOS) transmission having the frequency range of $10 \mathrm{GHz}$ to $66 \mathrm{GHz}$. The very critical element of WiMAX is orthogonal fre-

${ }^{*}$ Author for correspondence 
quency division multiple access. It is a multiple access scheme which supports many users at a time and hence leads to bandwidth efficiency of the system. It provides high-speed communication as well as removes intersymbol interference and inter-carrier interference at the same time. It is a multi-carrier communication where the wideband carrier is subdivided into subcarriers which are orthogonal to each other. It leads to better bandwidth utilization and can be implemented using signal processing block like Fast Fourier Transform (FFT) and Inverse Fast Fourier Transform (IFFT) ${ }^{2}$. WiMAX has a wonderful Quality Of Service (QoS) even in adverse channel condition plus it is possible to use multiple antennas at transmitter and receiver to provide diversity to the system $^{3}$. WiMAX offers high throughput for broadband access and hotspot users. It supports large features like adaptive modulations, adaptive antennas, multiple antennas, FDD, TDD and frequency reuse ${ }^{4}$. The channels used in this paper are fading channels which mean there is every chance that at some points of time the fading can be large and at some points fading can be small. In the case of large fading time or duration burst errors occurs, the codes which are very effective in detecting and correcting the burst errors are Reed-Solomon codes. They are nonbinary codes which have very large minimum distance and hence greater error correction capability. Also, these codes do not take in bits of data, but symbols of data and the redundancy added is also as symbols ${ }^{5}$. Another class of cyclic codes can also be used with WiMAX system to enhance its performance; those are $\mathrm{BCH}$ codes. These codes are generated using generator polynomials with the degree (n-k), where ' $n$ ' is the codeword length, and ' $k$ ' is the information word length. These codes have high coding gain and impressively high implementation speed ${ }^{6}$. Turbo codes can be very well implemented with WiMAX because they are capable of achieving Shannon's capacity. They are a combination of recursive systematic convolutional encoders and a random interleaver between them. They have the advantage that at lower SNR values they perform very well and in a way are a better version of convolutional codes ${ }^{7,8}$. Low-Density Parity-Check (LDPC) codes when used in Orthogonal Frequency Division Multiplexing (OFDM) system improves its performance, and these codes perform equally well with WiMAX system because OFDM is an integral part of it. LDPC codes have been recently redeveloped by Neil and McKay and have been evaluated for their performance. They perform much better compared to other block codes, and their idea was initially given in previous literature ${ }^{9,10}$. In years to come, $4 \mathrm{G}$ technology will over power all the currently working technologies and is supposed to join various wireless technologies to provide a better quality of service and meet the demand of the end users, WiMAX being an important part of this integration ${ }^{11-13}$.

\section{Model Description of Wimax Physical Layer}

The WiMAX physical layer was initially able to support only LOS transmissions for (10-66) GHz, and this required an antenna to be fixed at a place where we want the connection, and the antenna should have a fairly strong link for data, voice and video transfer. With time NLOS transmission was needed and then WiMAX physical layer was upgraded to support NLOS connections too for (2-11) GHz. With this update, we have WiMAX system physical layer that operates for both long and short distances. The basic block diagram of physical layer of WiMAX is given in Figure 1.

\subsection{Randomizer}

Randomizer scrambles the data coming to it from the higher layer (MAC) and is implemented using Pseudo Random Bit Generators or Linear Feedback Shift Registers. Randomization is done on bit by bit basis so that there is an uncertain distribution of zeroes and ones in the information word. Its main function is to improve the performance of the FEC block. At the receiver side, the scrambled data is descrambled and fed to the higher layer for further processing.

\subsection{FEC Encoder}

FEC block is the most important block of this physical layer. It adds redundancy in a controlled manner so that reliability of data is improved at the cost of greater bandwidth requirement. At the receiver, this redundancy is examined to see if there are any errors and if they are capable of correction. The redundancy at the receiver is removed. There are many FEC codes used since the past, but the ones used in this paper are explained briefly as:

1. BCH codes- They belong to a class of cyclic codes which are binary. Cyclic codes have certain properties which are also fulfilled by $\mathrm{BCH}$ codes. These codes have high coding gains. They can be designed to correct a specific number of errors, unlike other coding 
techniques which have fixed minimum distance and can correct a particular number of errors. They are developed from a generator polynomial, and they must have a primitive element. The primitive element and generator polynomial belong to different fields. These codes use syndrome decoding which is far easy to implement and reduces the cost of implementation. 2. RS codes- They are also a class of cyclic codes but are non-binary in nature. Being nonbinary, the range of errors it can correct is also large. It takes data as symbols, not in bit format. Also, it adds redundancy in symbol form than bit form. They were largely used in space communication and storage devices but are now widely used in wireless communication. These codes have the capability to correct long sequence of errors called burst errors.

3. Turbo codes- Turbo codes use two Recursive Systematic Convolutional (RSC) encoders and a parallel concatenation of these two with the random interleaver. The two encoders work collaboratively such that first RSC produces parity bits which are of low weight and the second RSC produces parity bits that are of high weight. Two different weighted parity bits are produced as a result of interleaver. A rate 1/3 turbo encoder is shown in Figure 2.

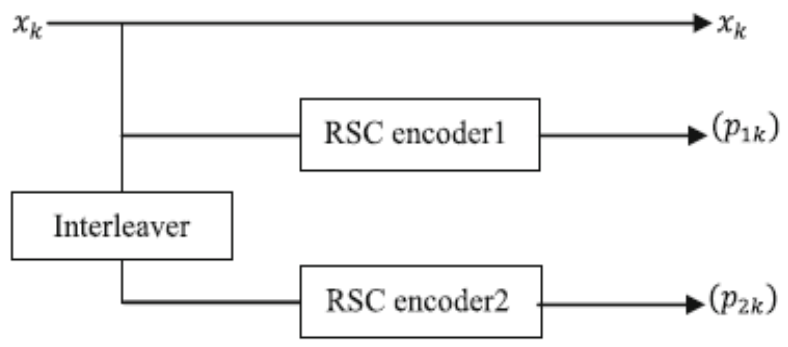

Figure 2. Rate 1/3 Turbo encoder

4. LDPC Codes- These codes were developed by R. Gallager in 1960s but were not appreciated much at that time because of the complexity of implementation and little development in the field of graph theory but recently they been acknowledged by many for their great performance. They have been used for Digital Video Broadcasting (DVB) based on satellites successfully and nowadays they are used with WiMAX. They are called low density because of the nature of their parity check matrix which is sparse. The parity check matrix can also be represented in the form of a Tanner graph. Tanner graph is a bipartite graph having two different types of nodes- check nodes and bit nodes. They can be decoded with ease and have the performance comparable to Shannon's limit.

\subsection{Interleaver}

Interleaver block is used to permute the order of bits so as to deal with the large sequence of consecutive errors. They are of many types, but the one used in this paper is block interleaver. At the receiver we user a similar deinterleaver block to undo the changes done by interleaver and retrieve the actual information. Randomizer, FEC block, and interleaver mostly work together to enhance the performance of channel coding.

\subsection{Modulator}

WiMAX uses adaptive modulation that is, the modulation changes depending on the channel conditions. When the channel quality is good, and there is the possibility of less signal degradation, higher order modulation is used, and when the channel quality is bad, and the signal is susceptible to large signal degradation, lower order modulation is used. This adaptive modulation scheme in WiMAX distinguishes it from other mobile communication systems. In this paper PSK modulation with orders 1 , 2,4 and 6 is used in system analysis.

\subsection{OFDM Multiplexer}

OFDM multiplexer has the function of constructing OFDM symbols by applying IDFT on the transmitter side whereas at the receiver side this process is reversed by used DFT. IDFT is a signal processing element that converts frequency domain signals to time domain signals and adds a guard band to prevent intermixing of signal or sub-carriers. These sub-carriers are perpendicular to each other preventing ICI and ISI. The OFDM symbol is shown in Figure 3. In this figure ' $\mathrm{T}$ ' represents the cyclic prefix appended, ' $\mathrm{T}_{\mathrm{b}}$ ' is the useful symbol time and ' $\mathrm{T}_{\mathrm{s}}$ ' is the total OFDM symbol time duration.

\subsection{Channel}

The channel is the interface between the transmitter and the receiver. Channels used in this paper are Rayleigh and Rician channels which are both fading channels. Fading occurs because signal takes different paths to travel from source to destination and there they combine constructively or destructively to cause fading. In dense areas 
there is no line of sight between the transmitter and the receiver and hence the approximation taken is Rayleigh fading distribution. In sparse areas, there is a dominant line of sight component stronger than other components which lead to rician fading approximation. The difference in rician channel and Rayleigh channel is that of $\mathrm{K}$ - factor. When $\mathrm{K}=0$, its Rayleigh fading channel and when $\mathrm{K}>0$, it's a rician fading channel. Mathematically,

$$
k=\frac{s^{2}}{2 \sigma^{2}}
$$

Where, 's' is the power of LOS component and ' $\sigma$ ' ' is the power in the NLOS components.

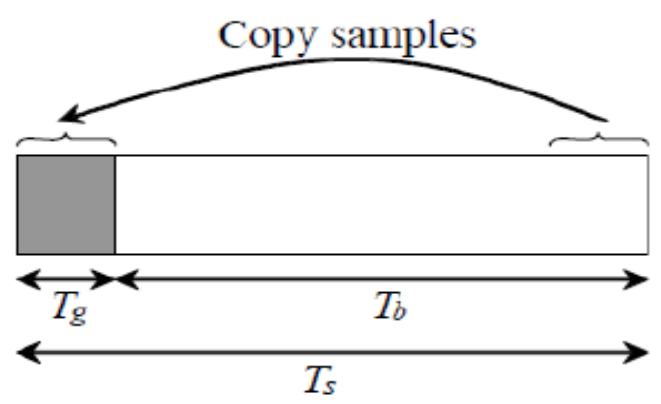

Figure 3. OFDM symbol format

\section{Results Discussion}

The simulation results consist of BER versus SNR graphs of WiMAX system for the two fading channels, taken over MPSK and with four different FEC techniques. Some light will be thrown on each of this one by one. The performance of a WiMAX system over Rayleigh and Rician channel based on BCH codes and using BPSK modulation is shown in Figure 4(a). Analyzing this figure we see that a BER of $10^{-3}$ is achieved in the rician channel at an SNR of $5.8 \mathrm{~dB}$ where as in the case of Rayleigh channel same is obtained at an SNR of 9.5dB. Rician channel requires $3.7 \mathrm{~dB}$ less SNR for same BER value and hence performs better. Taking a look at the next figure using Quadrature Phase Shift Keying (QPSK) in BCH-based WiMAX, we see that BER of $10^{-3}$ is achieved by the rician channel at $5.9 \mathrm{~dB}$ and Rayleigh channel at $8.1 \mathrm{~dB}$. So Figure 4(b) shows an SNR improvement of $2.2 \mathrm{~dB}$ and out performs Rayleigh channel. Taking a look at the next figure in which other parameters are same, but modulation is 16 -PSK, we see that $10^{-3}$ BER is achieved at SNR of $7.8 \mathrm{~dB}$ in rician channel and at $13.6 \mathrm{~dB}$ in Rayleigh channel as presented in Figure 4(c). So rician channel provides better performance compared to Rayleigh channel by utilizing $5.8 \mathrm{~dB}$ less SNR. The other figure of WiMAX based on $\mathrm{BCH}$ codes is modulated by 64-PSK shown in Figure 4(d). A careful study shows that rician channel needs $5.4 \mathrm{~dB}$ less SNR for a BER of $10^{-3}$. For this BER rician channel necessities $15 \mathrm{~dB}$ SNR value and Rayleigh channel necessities 20.4dB SNR value.

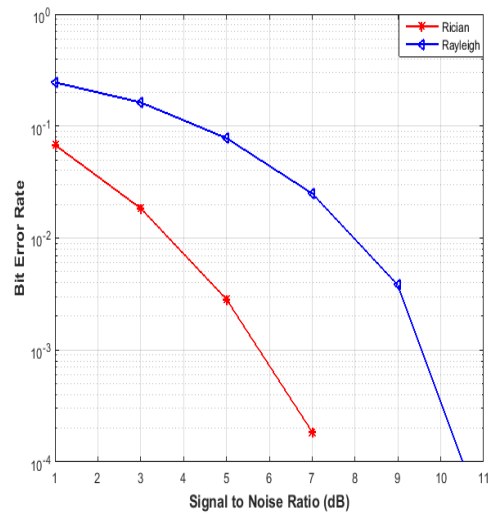

(a)

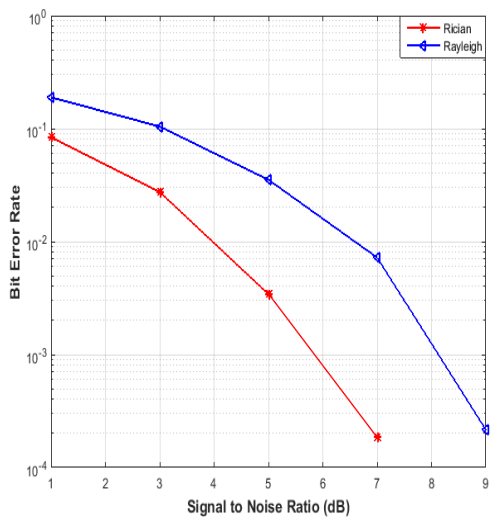

(b)

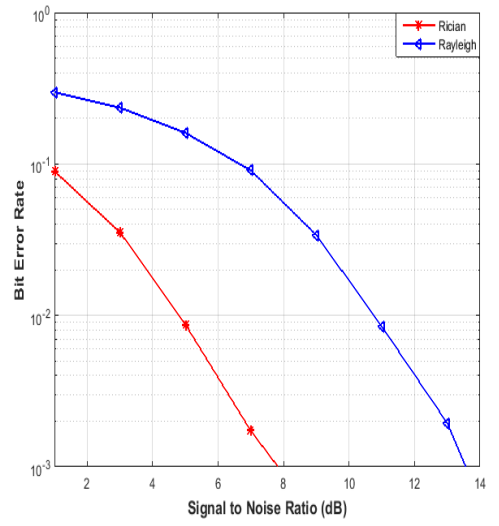

(c) 


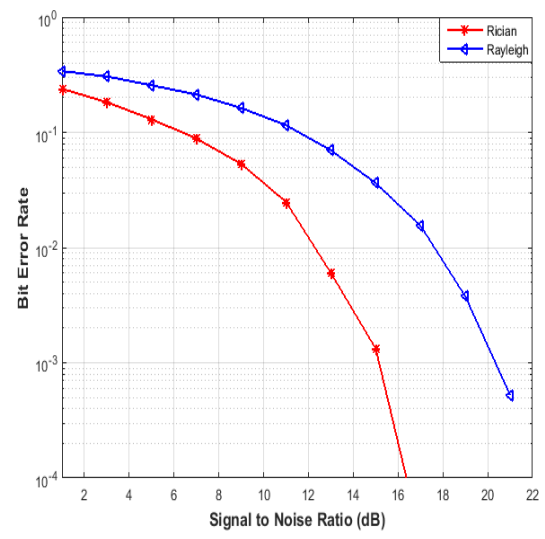

(d)

Figure 4. (a-d). SNR vs. BER Comparison for WiMAX Based on BCH Codes over Diverse Fading Channels (a) BPSK, (b) QPSK, (c) 16-PSK, and (d) 64-PSK

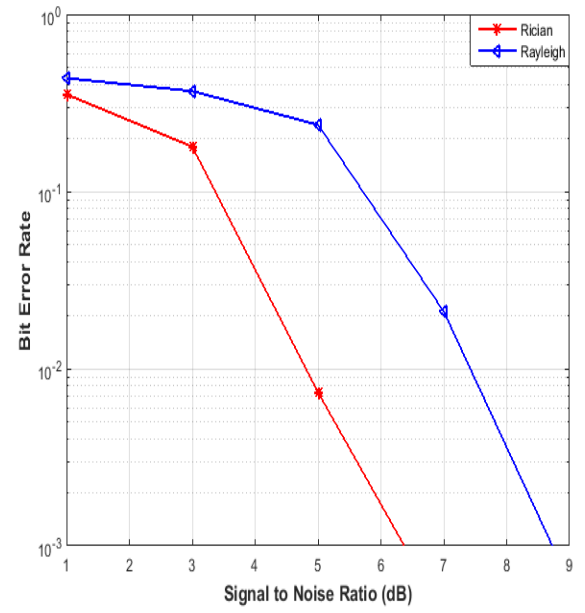

(a)

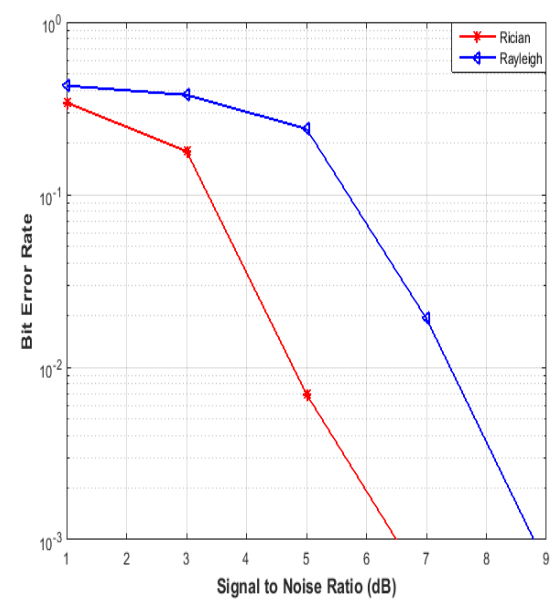

(b)

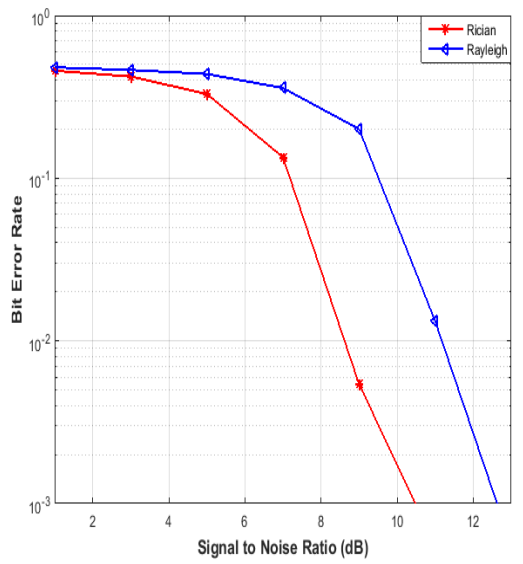

(c)

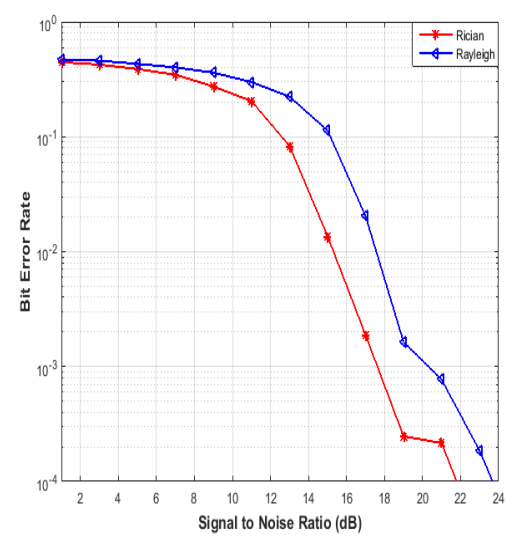

(d)

Figure 5. (a-d) SNR vs. BER Comparison for WiMAX Based on Turbo Codes over Diverse Fading Channels (a) BPSK, (b) QPSK, (c) 16-PSK, and (d) 64-PSK

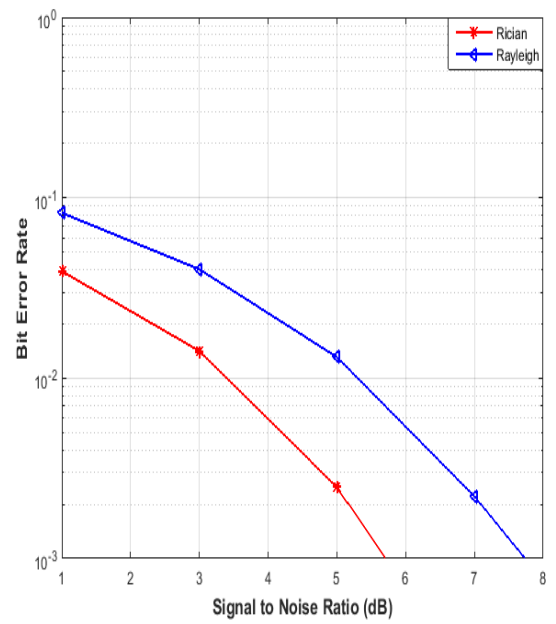

(a) 


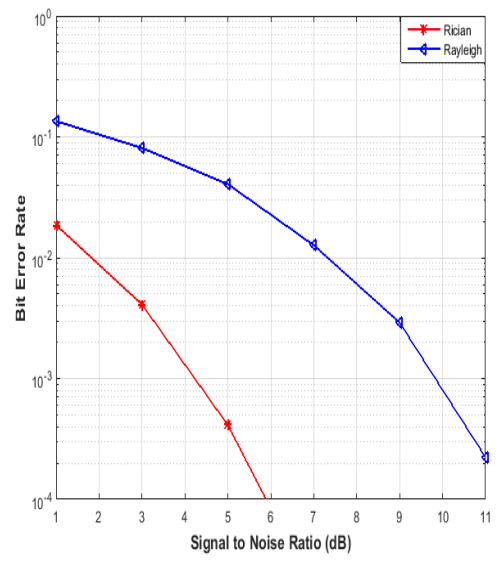

(b)

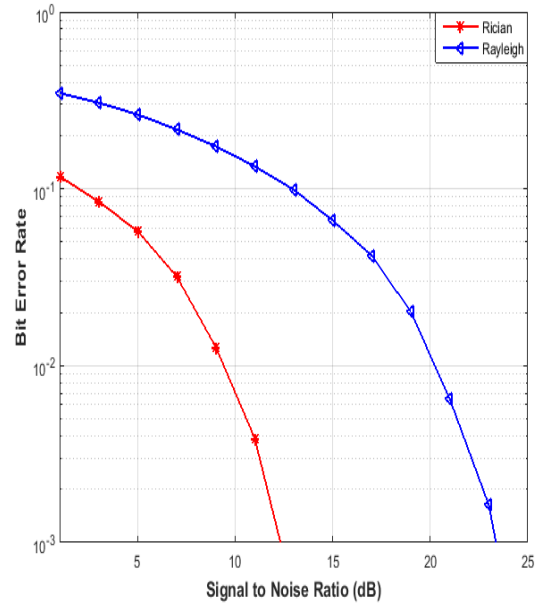

(c)

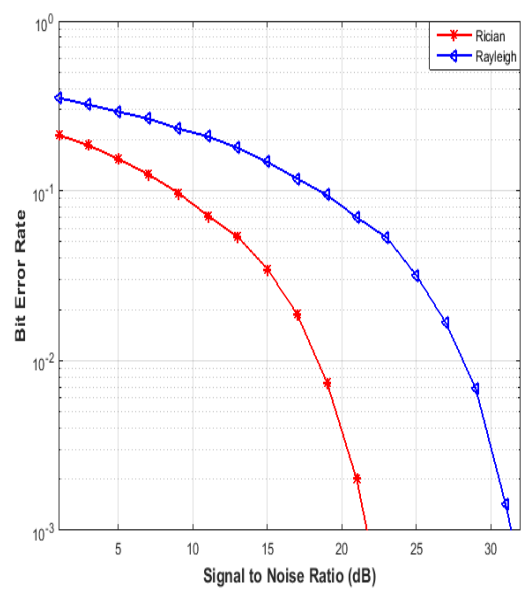

(d)

Figure 6. (a-d) SNR vs. BER Comparison for WiMAX Based on LDPC Codes over Diverse Fading Channels (a) BPSK, (b) QPSK, (c) 16-PSK, and (d) 64-PSK

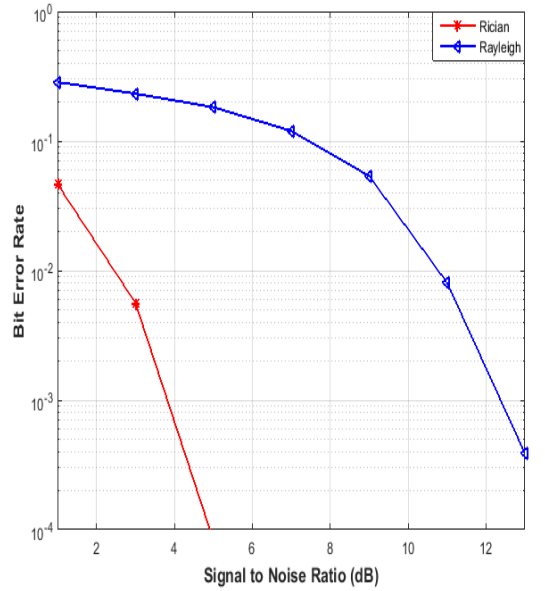

(a)

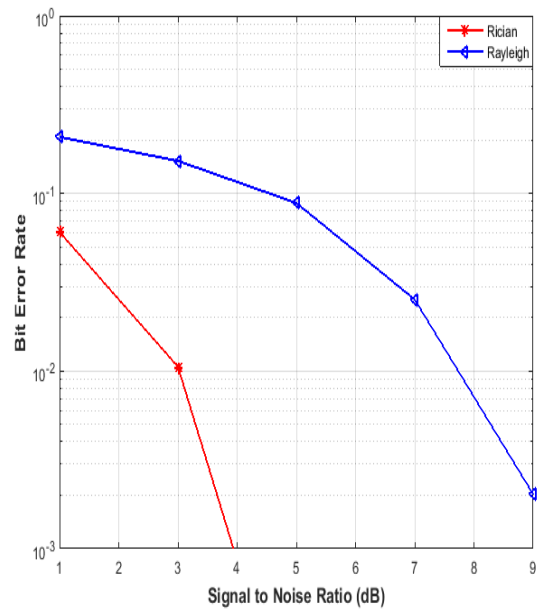

(b)

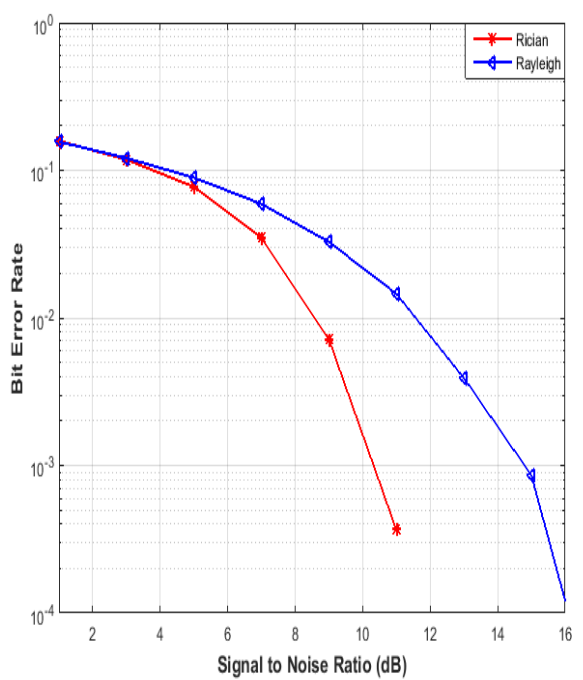

(c) 


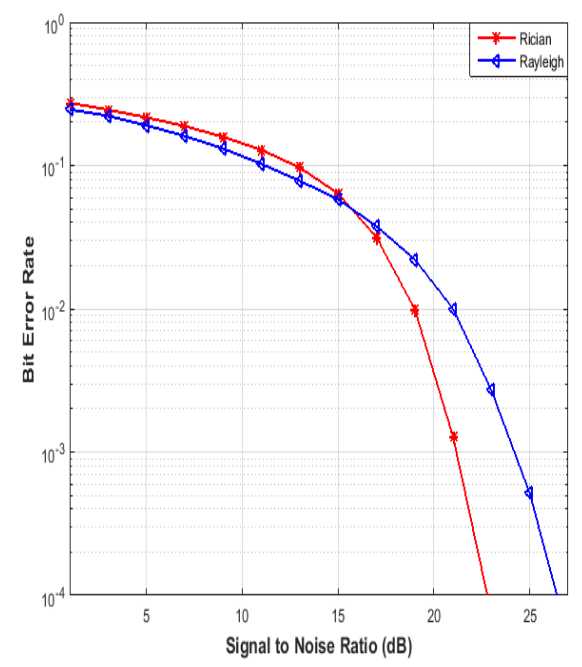

(d)

Figure 7. (a-d) SNR vs. BER Comparison for WiMAX Based on RS Codes over Diverse Fading Channels (a) BPSK, (b) QPSK, (c) 16-PSK, and (d) 64-PSK

For BPSK modulation, rician channel requires an SNR of $6.4 \mathrm{~dB}$ and Rayleigh channel requires an SNR of $8.7 \mathrm{~dB}$ as shown in Figure 5(a).The difference in SNR is about $2.3 \mathrm{~dB}$ which denotes rician channel has better performance. In the following Figure 5(b), the modulation order is changed to 4 and the examined results show that for a BER of $10^{-3}$, rician channel needs an SNR of $6.5 \mathrm{~dB}$ and Rayleigh channel must have an SNR of $8.8 \mathrm{~dB}$. Again rician channel requires $2.3 \mathrm{~dB}$ lesser SNR to achieve same BER and hence performs better. Considering Figure 5(c), the modulation used is 16-PSK and the error rate of $10^{-3}$ is achieved by the rician channel at $10.4 \mathrm{~dB}$ and by Rayleigh channel at $12.5 \mathrm{~dB}$. The SNR improvement shown by rician channel is $2.1 \mathrm{~dB}$. The other figure uses the same system with modulation of 64-PSK. Given Figure 5(d), SNR required by rician channel for a $\mathrm{BER}$ of $10^{-3}$ is $17.5 \mathrm{~dB}$ and SNR required by Rayleigh channel is $20 \mathrm{~dB}$, presenting an improvement of $2.5 \mathrm{~dB}$ in SNR for rician channel with respect to Rayleigh channel. The performance of a WiMAX system over Rayleigh and Rician channel based on LDPC codes and using BPSK modulation is shown in Figure 6(a). Analyzing this figure we see that a BER of $10^{-3}$ is achieved in the rician channel at an SNR of $5.7 \mathrm{~dB}$ where as in the case of Rayleigh channel same, is obtained at an SNR of 7.8dB. Rician channel requires $2.1 \mathrm{~dB}$ less SNR for same BER value and hence performs better. Taking a look at the next figure using QPSK in LDPC based WiMAX, we see that BER of $10^{-3}$ is achieved by rician channel at $4.2 \mathrm{~dB}$ and Rayleigh channel at 9.9dB. So Figure 6(b) shows an SNR improvement of $5.7 \mathrm{~dB}$ and out performs Rayleigh channel. Taking a look at the next figure in which other parameters are same but modulation is 16-PSK, we see that $10^{-3} \mathrm{BER}$ is achieved at SNR of $12.2 \mathrm{~dB}$ in the rician channel and at $23 \mathrm{~dB}$ in Rayleigh channel as presented in Figure 6(c). So the rician channel provides better performance compared to Rayleigh channel by utilizing $10.8 \mathrm{~dB}$ less SNR. The other figure of WiMAX based on LDPC codes is modulated by 64-PSK shown in Figure 6(d). A careful study shows that rician channel needs $9.6 \mathrm{~dB}$ less SNR for a BER of $10^{-3}$. For this BER rician channel necessities 21.9dB SNR value and Rayleigh channel necessities $31.5 \mathrm{~dB}$ SNR value. For the other four figures, the channel coding used is RS code, and the channels of the WiMAX system are the same. For BPSK modulation, rician channel requires an SNR of 3.8dB and Rayleigh channel requires an SNR of $12.6 \mathrm{~dB}$ as shown in Figure 7(a).The difference in SNR is about $8.8 \mathrm{~dB}$ which denotes rician channel has better performance. In the following Figure 7(b), the modulation order is changed to 4 and the examined results show that for a BER of $10^{-3}$, the rician channel needs an SNR of 3.9dB and Rayleigh channel must have an SNR of $9 \mathrm{~dB}$. Rician channel requires $5.1 \mathrm{~dB}$ lesser SNR to achieve same BER and hence performs better. Considering Figure 7 (c), the modulation used is 16-PSK and the error rate of $10^{-3}$ is achieved by the rician channel at $10.5 \mathrm{~dB}$ and by Rayleigh channel at $15 \mathrm{~dB}$. The SNR improvement shown by the rician channel is $4.5 \mathrm{~dB}$. The other figure uses the same system based on RS codes with 64-PSK modulation. In view of Figure 7(d), SNR required by the rician channel for a BER of $10^{-3}$ is $23.5 \mathrm{~dB}$ and SNR required by Rayleigh channel is $27 \mathrm{~dB}$, presenting an improvement of $3.5 \mathrm{~dB}$ in SNR for the rician channel with respect to Rayleigh channel. The complete summary of the results is presented in Table 1.

\section{Conclusion}

With the results shown previously, the conclusion reached is that for a WiMAX system based on $\mathrm{BCH}$ codes where channels are rician and Rayleigh the improvement in SNR value for these codes range between $2.2 \mathrm{~dB}$ and $5.8 \mathrm{~dB}$ for different modulation orders of PSK. When the same system is based on turbo codes the improvement in rician channel for the same is between $2.1 \mathrm{~dB}$ and $2.5 \mathrm{~dB}$ 
Table 1. SNR Requirement in WiMAX for Diverse Channel Encoding Schemes

\begin{tabular}{|l|l|l|l|l|l|l|l|l|}
\hline \multirow{3}{*}{ Modulation } & \multicolumn{9}{l}{ SNR(dB) required to achieve a BER of $10^{-3}$} \\
\cline { 2 - 10 } & \multicolumn{3}{|l|}{ Rician Channel } & \multicolumn{3}{l|}{ Rayleigh Channel } \\
\cline { 2 - 10 } & BCH & LDPC & RS & TURBO & BCH & LDPC & RS & TURBO \\
\hline BPSK & 5.8 & 5.7 & 3.8 & 6.4 & 9.5 & 7.8 & 12.6 & 8.7 \\
\hline QPSK & 5.9 & 4.2 & 3.9 & 6.5 & 8.1 & 9.9 & 9 & 8.8 \\
\hline 16-PSK & 7.8 & 12.2 & 10.5 & 10.4 & 13.6 & 23 & 15 & 12.5 \\
\hline 64-PSK & 15 & 21.9 & 23.5 & 17.5 & 20.4 & 31.5 & 27 & 20 \\
\hline
\end{tabular}

for MPSK modulation. If WiMAX system is using LDPC codes for error correction and the channels are the fading ones with PSK modulation of different orders, rician channel performs much better than Rayleigh channel with the improvement of SNR value lying between $2.1 \mathrm{~dB}$ and $10.8 \mathrm{~dB}$. The last case considers the WiMAX system based on RS codes and the fading channels for transmission, where again rician channel performs better than Rayleigh channel with improvements in SNR values between $3.5 \mathrm{~dB}$ and $8.8 \mathrm{~dB}$. This leads to the verdict that in a WiMAX system rician channel performance is better using any FEC technique and with any modulation scheme and should be preferred over Rayleigh channel.

\section{References}

1. Sedani B.S., Kotak N.A., Borisagar K.R., Kulkarni G.K. Implementation and Performance Analysis of Efficient Wireless Channels in WiMAX using Image and Signal Transmission. In Proceedings of International Conference on Communication Systems and Network Technologies, 2012, 630-34.

2. Patidar M., Dubey R., Jain N.K., Kulpariya S. Performance Analysis of WiMAX e Physical Layer Model. In Proceedings of International Conference on Wireless and Optical Communication Networks, 2012, 802(16), 1-4.

3. Ahmadzadegan M.H., Elmusrati M., Widyotriatmo A. WiMAX Based on Energy Efficient Intrusion Detection System. In Proceedings of International Conference on Robotics, Biomimetics and Intelligent Computational Systems, 2013, 166-69.

4. Benmusa T.A., Belgassem A.J., Ibrahim M.A.S. Planning and Dimensioning High Speed 4G WiMAX Network in
Tripoli Area. In Proceedings of International Conference on Science and Techniques of Automatic Control and Computer Engineering, 2013, 318-24.

5. Logeshwaran R., Paul I.J.L . Performance study on the Suitability of Reed Solomon Codes in WiMAX. In Proceedings of Internal Conference on Wireless Communication and Sensor Computing, 2010, 1-4 .

6. Rappaport T.S. Wireless Communications Principles and Practice. Second Edition, Pretence Hall. 2002; 1-7.

7. Xinyu Z. A Basic Research on Forward Error Correction. In Proceedings of International Conference on Communication Software and Networks, 2011, 462-65.

8. Rao K.D. Channel Coding Techniques for Wireless Communication, Springer, 2015.

9. Benjangkaprasert C., Inchan T. Signal Processing of WiMAX Physical Layer Based on Low-Density Parity-Check Codes. In the Proceedings of International Conference on Control Automation and Systems, 2014, 556-60 .

10. Png K.B., Peng X., Chin F. Performance Studies of a Multiband OFDM System Using Simplified LDPC Code. In Proceedings of International Conference on Ultrawideband Systems and Technologies, 2004, 376-80.

11. Khan S., Fisal N., Bakar Z.A., Salawu N., Maqbool W., Ullah R., Safdar H. Secure Authentication and Key Management Protocols for Mobile Multihop WiMAX Networks. Indian Journal of Science and Technology. 2014 Jan; 7(3):282-95.

12. Singh C., Patterh M.S., Sharma S. Design of Programmable Digital down Converter for WiMAX. Indian Journal of Science and Technology. 2009 Mar; 2(3):1-2.

13. Qamar R.A., Maarof M.A., Ibrahim S. An Efficient Encoding Algorithm for (n, k) Binary Cyclic Codes. Indian Journal of Science and Technology. 2012 May; 5(5):1-5. 